\title{
Theoretical Study of the Methanol Dehydrogenation on Platinum Nanocluster
}

\author{
Svetlana S. Laletina ${ }^{a}$, Elena A. Shor*a, \\ Mikhail I. Mamatkulovb, Ilya V. Yudanov', \\ Vasily V. Kaichev ${ }^{\mathbf{b}}$ and Valerii I. Bukhtiyarov ${ }^{\mathbf{b}}$ \\ a Institute of Chemistry and Chemical Technology SB RAS \\ FRC "Krasnoyarsk Science Center SB RAS" \\ 50/24 Akademgorodok, Krasnoyarsk, 660036, Russia \\ ${ }^{b}$ Boreskov Institute of Catalysis, SB RAS \\ 5 Akademika Lavrentieva, Novosibirsk, 630090, Russia
}

Received 27.07.2016, received in revised form 14.10.2016, accepted 09.12.2016

The methanol dehydrogenation through the initial breaking of the $\mathrm{O}-\mathrm{H}$ bond at $P \mathrm{t}_{79}$ nanoparticle was studied with the DFT method. The comparison with an ideal surface of Pt (111) was carried out. The most stable complexes were found for $\mathrm{COH}_{x}(x=1-4)$ species adsorbed at low-coordinated atoms of nanocluster $P t_{79}$, whereas no preference for adsorption at corners and edges for $H$ atoms was found. The absolute adsorption energies of $\mathrm{COH}_{x}$ species at corner and edge sites of platinum nanocluster increased by 0.2-0.7 eV in comparison with high-coordinated sites of the regular Pt(111) surface. The stabilization effect of adsorption at the nanoparticle decreases from corners to edges and then to the center of (111) facet. According to the reaction energy profile, the thermal effect of the formation of $\mathrm{CH}_{3} \mathrm{O}$ at the nanocluster becomes close to zero, in contrast to the endothermic effect $(0.5 \mathrm{eV})$ on the regular Pt(111) surface. The exothermic effects for other reaction stages at the platinum nanocluster, excluding $\mathrm{CO}$ desorption, increase by $\sim 0.2-0.5 \mathrm{eV}$.

Keywords: DFTmethod, methanol, dehydrogenation, intermediates, Ptnanoparticles, low-coordinated centers.

DOI: $10.17516 / 1998-2836-2016-9-4-430-442$.

(C) Siberian Federal University. All rights reserved

* Corresponding author E-mail address: sekr@icct.ru 


\title{
Теоретическое исследование дегидрирования метанола
}

\section{на нанокластере платины}

\author{
С.С. Лалетина ${ }^{a}$, Е.А. Шора, М.И. Маматкулов ${ }^{\sigma}$, \\ И.В. Юданов ${ }^{\tilde{}}$, В.В. Каичев ${ }^{\tilde{0}}$, В.И. Бухтияров ${ }^{\sigma}$ \\ ${ }^{a}$ Институт химии и химической технологии СО РАН \\ ФИЦ «Красноярский научный центр СО РАН» \\ Россия, 660036, Красноярск, Академгородок, 50/24 \\ ${ }^{6}$ Институт катализа им. Г.К. Борескова СО РАН \\ Россия, 630090, Новосибирск, пр. Академика Лаврентьева, 5
}

Методом функционала плотности изучена реакция дегидрирования метанола по механизму разрыва О-Н-связи на нанокластере платины $P t_{79}$, проведено сравнение с идеальной поверхностью Рt(111). Найдено, что наиболее устойчивые комплексы образуются при адсорбции $\mathrm{COH}_{x}(x=1-4)$ частии на низкокоординированных атомах нанокластера $P t_{79}$, при этом такой предпочтительности для атомов Н не обнаружено. Абсолютные значения энергии адсорбиии на вершинах и ребрах нанокластера $\mathrm{Pt}_{79}$ выше на 0,2-0,7 эВ, чем на высококоординированных цุентрах регулярной поверхности Рt(111). Стабильность адсорбционных комплексов на поверхности нанокластера уменьшается от вершин к ребрам и затем к иентру граней (111) нанокластера. Анализ энергетического профиля реакции показывает, что тепловой эффект образования ключевого интермедиата $\mathrm{CH}_{3} \mathrm{O}$ на кластере $\mathrm{Pt}_{79}$ становится нулевым в отличие от эндотермического (0,5 эB) на регулярной поверхности Pt(111). Экзотермический эффект всех остальных реакциионных стадий, за исключением десорбции СО, на нанокластере увеличивается на 0,2-0,5 эB.

Ключевые слова: метод функционала плотности, метанол, дегидрирование, интермедиатьи, наночастицы Pt, низкокоординированные центры.

\section{Введение}

Метанол является дешевым и доступным сырьем, используемым в различных областях промышленности. В частности, в последние годы метанол рассматривается как сырье для производства водорода в малогабаритных каталитических конверторах [1]. Для получения водорода можно использовать процесс дегидрирования метанола до СО и водорода либо селективное окисление метанола до $\mathrm{CO}_{2}$ и $\mathrm{H}_{2}$. Оба процесса с высокой эффективностью происходят на платиновых катализаторах. Многочисленные экспериментальные [2-12] и теоретические [13-21] исследования механизмов разложения и окисления метанола на поверхности платины выявили три основных пути протекания реакций: через первоначальный разрыв О-Н-связи и образование метоксигруппы $\mathrm{CH}_{3} \mathrm{O}(1)$, через разрыв связи $\mathrm{C}-\mathrm{H}$ и образование гидроксиметиленовой группы $\mathrm{CH}_{2}-\mathrm{OH}$ (2) или же через разложение метанола с разрывом С-O связи (3). В первых двух случаях происходит полное дегидрирование метанола до $\mathrm{H}_{2}$ и $\mathrm{CO}$, в последнем - неполное 
дегидрирование до $\mathrm{H}_{2}$ и углеродсодержащих частиц $\mathrm{CH}_{x}$. Третий механизм реакции нежелателен, так как приводит к зауглероживанию поверхности платины, что препятствует адсорбции реагентов и существенно снижает скорость процесса дегидрирования. Поэтому выявление факторов, способствующих селективному протеканию реакции по первым двум маршрутам, представляет практический интерес и является одной из основных задач при дальнейшем усовершенствовании платиновых катализаторов.

Недавно было установлено, что морфология поверхности и размер нанесенных частиц платины влияет на пути трансформации молекул метанола и селективность по основным продуктам каталитических превращений. Так, например, методом температурно-программируемой реакции было показано, что при окислении метанола на дефектной поверхности платины (111) наблюдается более высокая селективность образования СО по сравнению с гладкой поверхностью $\mathrm{Pt}(111)$ [22]. Исследования электрохимическими методами с привлечением расчетов методом функционала плотности $[20,23,24]$ подтвердили, что окислительное дегидрирование метанола на дефектных поверхностях платины предпочтительно протекает через разрыв О-Нсвязи и последующий отрыв атомов водорода:

$$
\mathrm{CH}_{3} \mathrm{OH}>\mathrm{CH}_{3} \mathrm{O}>\mathrm{CH}_{2} \mathrm{O}>\mathrm{CHO}>\mathrm{CO} \text {. }
$$

Наличие высоко- (ВК) и низкокоординированных (НК) атомов платины, расположенных на наногранях, ребрах и вершинах нанокластеров, делают эти системы также привлекательными для проведения реакции разложения метанола. Так, на наночастицах платины размером 23-13 нм $[25,26]$ наблюдалось исключительно образование $\mathrm{CO}$ и $\mathrm{H}_{2}$ в качестве продуктов конверсии метанола. Теоретические расчеты реакции разложения метанола на частицах платины ограничены изучением субнаноразмерных моделей $\mathrm{Pt}_{8}$ [13] и $\mathrm{Pt}_{10}$ [14]. Поскольку протекание реакции на наночастицах платины более крупного размера (1 нм и более) до сих пор не рассматривалось теоретическими методами, целью данной работы явилось квантово-химическое исследование реакции разложения метанола на нанокластере платины $\mathrm{Pt}_{79}$. Для сравнения аналогичные расчеты проведены с использованием модели кристаллических пластин (слэб, slab), имитирующих поверхность монокристалла $\mathrm{Pt}(111)$.

\section{Модели и детали расчета}

Периодические расчеты электронных структур выполнялись методом функционала плотности с использованием обобщенного градиентного приближения [27] и псевдопотенциала в параметризации Пердью-Ванга'91 (энергия отсечки 400 эВ) в программном пакете VASP [28, 29]. Все рассматриваемые адсорбционные комплексы на поверхности металла и металлической наночастицы имеют закрытые электронные оболочки. Влияние остовных электронов на валентную плотность учитывалось с помощью метода PAW (projector augmented wave method) $[30,31]$, обеспечивающего сходимость энергий до значений в $10^{-6}$ эВ. Частичная заселенность волновой функции достигалась при помощи уширения Метфесселя-Пакстона второго порядка с величиной 0,1 эВ. При оптимизации “объемной” платины (атомов платины, входящих в состав ядра кластера и в состав используемых слэбов периодической модели) использовалась сетка Монхорста-Пака размерностью $(13 \times 13 \times 13)$ [32]. При оптимизации поверхностей и кластера применялись $\mathrm{G}$-центрированные автоматические сетки $k$-точек $4 \times 4 \times 1$ и $1 \times 1 \times 1$ соответствен- 
но. Оптимизация положений атомов проводилась до достижения величины градиентов менее 0,01 эB/Å.

В качестве одной из моделей поверхности платины использовалась пятислойная периодическая 2D-пластина, имитирующая регулярную поверхность $\operatorname{Pt}(111)$ с элементарной ячейкой размера $3 \times 3$, состоящей из 45 атомов (рис. 1 a). Пятислойные пластины имели толщину $\sim 9 \AA$ и были разделены вакуумным пространством в $18 \AA$. Нижние три слоя пластины (27 атомов) фиксировались в положениях, соответствующих объемной структуре, а два верхних (18 атомов) - оптимизировались. Моделировалось низкое молекулярное покрытие поверхности 1/9 монослоя (МС), соответствующее одной молекуле адсорбата на элементарную ячейку.

В качестве альтернативной модели поверхности платинового катализатора был выбран октаэдрический кластер $\mathrm{Pt}_{79}$ с диаметром $\sim 1,14$ нм, на поверхности которого присутствуют высоко- (с координационным числом КЧ $=9$ ) и низкокоординированные (с КЧ $=6-7)$ атомы (рис. 16). Такие усеченные октаэдрические кластеры обеспечивают репрезентативное распределение центров адсорбции на наногранях (111) и (100), а также на углах и ребрах [33, 34]. Ранее аналогичная модель $\mathrm{Pd}_{79}$ была использована для исследования дегидрирования метанола на наночастицах $\mathrm{Pd}$ [35]. Для исключения взаимодействий между кластерами в расчетах применялась периодическая ячейка размером $34 \times 34 \times 34 \AA$. Таким образом, кластеры были разделены вакуумным пространством в $23 \AA$.

Энергии адсорбции $E_{a d s}$ были рассчитаны согласно уравнению (1) вычитанием свободной энергии молекулы адсорбата $\left(E_{a d s}\right)$ и энергии субстрата $\left(E_{s u b}\right)$ из полной энергии комплекса $\left(E_{a d s / s u b}\right)$ :

$$
\mathrm{E}_{\mathrm{ads}}=\mathrm{E}_{\mathrm{ads} / \mathrm{sub}}-\mathrm{E}_{\mathrm{ads}}-\mathrm{E}_{\mathrm{sub}} .
$$

Энергии реакций элементарных стадий реакции дегидрирования метанола $E_{R}$ вычисляли по уравнению (2) вычитанием суммы энергий реагирующих веществ $\left(E_{\text {react }}\right)$ из суммарной энергии продуктов реакции $\left(E_{p r o d}\right)$ :

$$
\mathrm{E}_{\mathrm{R}}=\Sigma \mathrm{E}_{\text {prod }}-\Sigma \mathrm{E}_{\text {react }} .
$$

Отрицательные значения $E_{a d s}$ и $E_{R}$ соответствуют экзотермическим процессам. Значение $\mathrm{E}_{\mathrm{ads}}$ получено помещением частиц в центре элементарной ячейки с нарушенной симметрией размерами $20 \times 21 \times 22 \AA$, зона Бриллюэна дискретизирована в $k$-точке.

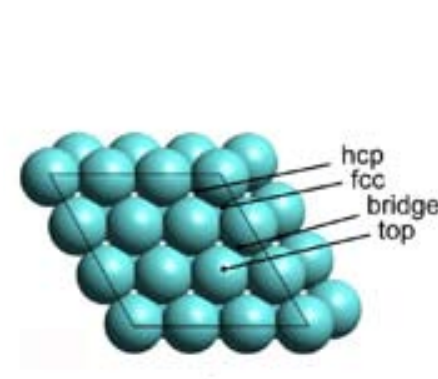

a

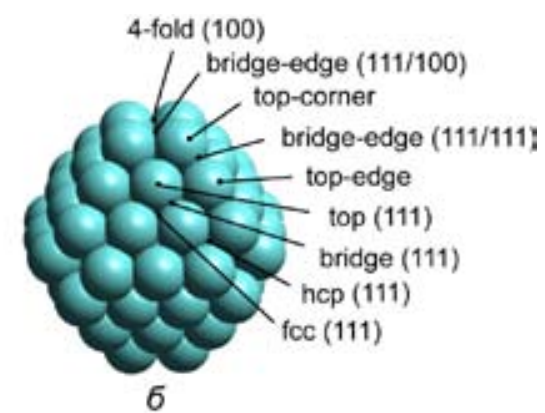

6

Рис. 1. Центры адсорбции поверхности $\mathrm{Pt}(111)$ (а) и нанокластера $\mathrm{Pt}_{79}$ (б)

$$
-433-
$$


Рассчитанная равновесная постоянная решетки для объемной платины равна $3,99 \AA$, что хорошо согласуется с экспериментальным значением $3,912 \AA$ [36].

\section{Результаты и обсуждение}

Для построения энергетического профиля реакции дегидрирования метанола была рассчитана адсорбция всех участвующих в реакции частиц в различных положениях идеальной поверхности $\operatorname{Pt}(111)$ и нанокластера $\mathrm{Pt}_{79}$. Обозначения адсорбционных центров платины и структуры поверхностных комплексов показаны на рис. 1 и 2 соответственно, энергии наиболее стабильных адсорбционных комплексов приведены в табл. 1. Наши результаты расчетов для поверхности $\operatorname{Pt}(111)$ сопоставлены с результатами более ранних теоретических работ [16, $17,20]$. Сначала будет рассмотрена адсорбция исходного вещества (метанол) и продукта реакции (СО), а затем - интермедиатов реакции.

\section{Структура и энергии адсорбиионных комплексов}

Метанол. На идеальной поверхности $\mathrm{Pt}(111) \mathrm{CH}_{3} \mathrm{OH}$ адсорбируется в тор-положении, координируясь к поверхностному атому Рt своим кислородным атомом (рис. 2). Рассчитанная нами энергия адсорбции, $-0,35$ эВ, хорошо согласуется с экспериментальным значением, $-0,48$ эВ, полученным с помощью метода температурно-программируемой десорбции (ТПД) [37] и теоретически рассчитанными энергиями, $-0,33$ [17, 20] и 0,46 эВ [16].

В различных top-положениях наночастицы $\mathrm{Pt}_{79}$ (в вершинах, на ребрах и гранях) метанол адсорбируется с энергиями в диапазоне -0,47-0,58 эВ, превышающими таковые для идеальной поверхности $\mathrm{Pt}(111)$. Так, адсорбция в наиболее выгодном положении на вершине нанокластера

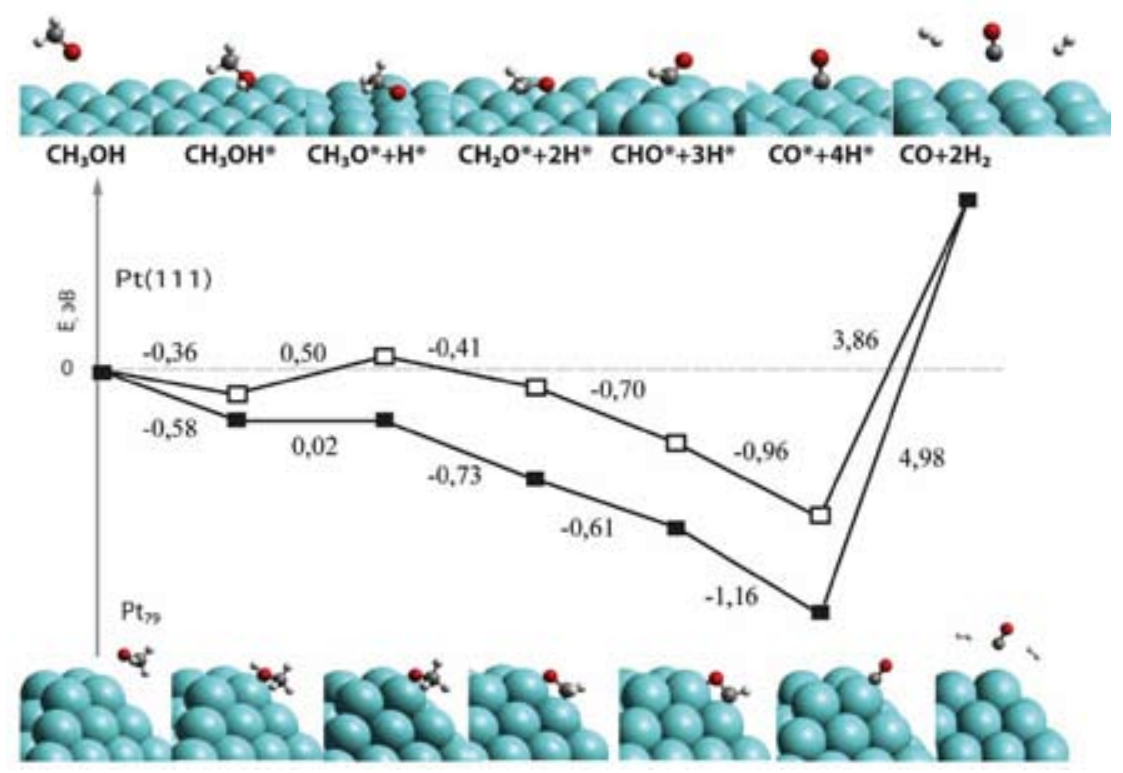

Рис. 2. Энергетический профиль реакции дегидрирования метанола на регулярной поверхности $\mathrm{Pt}(111)$ (белые квадраты) и кластере $\mathrm{Pt}_{79}$ (черные квадраты) и структуры наиболее стабильных интермедиатов реакции. Символ * обозначает адсорбцию на поверхности. Тепловые эффекты элементарных стадий даны в эВ 
Таблица 1. Энергии адсорбции (в эВ) интермедиатов реакции дегидрирования метанола на поверхности $\mathrm{Pt}(111)$ и нанокластера $\mathrm{Pt}_{79}$. Даны энергии для наиболее прочно связанных комплексов каждого типа. Структуры адсорбционных комплексов см. на рис. 2

\begin{tabular}{|c|c|c|c|c|c|}
\hline \multirow{2}{*}{ Интермедиаты } & \multicolumn{2}{|r|}{$\operatorname{Pt}(111)$} & \multicolumn{2}{|r|}{$\mathrm{Pt}_{79}$} & \multirow{2}{*}{$\Delta \mathbf{E}^{a}$} \\
\hline & $E_{\text {ads }}$ & ЦА ${ }^{\sigma}$ & $\overline{E_{\text {ads }}}$ & ЦА $^{\sigma}$ & \\
\hline $\mathrm{CH}_{3} \mathrm{OH}$ & $-0,35$ & top & $-0,58$ & top-corner & $-0,23$ \\
\hline $\mathrm{CH}_{3} \mathrm{O}$ & $-1,80$ & top & $-2,34$ & top-corner & $-0,54$ \\
\hline & $-1,75$ & bridge & $-2,37$ & bridge-edge $111 / 100$ & $-0,62$ \\
\hline $\mathrm{CH}_{2} \mathrm{O}$ & $-0,58$ & top-top & $\begin{array}{l}-1,28 \\
-1,20\end{array}$ & $\begin{array}{l}\text { top-corner }(O) \text {-top-edge }(C) \\
\text { top-corner }(C) \text {-top-edge }(O)\end{array}$ & $-0,70$ \\
\hline $\mathrm{CHO}$ & $\begin{array}{l}-2,52 \\
-2,46\end{array}$ & $\begin{array}{l}\text { top } \\
\text { top }(C) \text {-bridge }(O)\end{array}$ & $\begin{array}{l}-3,00 \\
-2,95 \\
-2,75\end{array}$ & $\begin{array}{l}\text { top-corner }(C) \text {-top-edge }(O) \\
\text { top-corner }(O) \text {-top-edge }(C) \\
\text { top-corner }(C)\end{array}$ & \begin{tabular}{|l|}
$-0,48$ \\
$-0,43$
\end{tabular} \\
\hline $\mathrm{CO}$ & $-1,71$ & top & $-2,28$ & bridge-edge & $-0,57$ \\
\hline & $-1,83$ & $f c c$ & $-2,19$ & top-corner & \\
\hline $\mathrm{H}$ & $-2,77$ & top & $-2,95$ & top (111) & \begin{tabular}{|l|}
$-0,18$ \\
\end{tabular} \\
\hline & & & $-2,95$ & bridge-edge & \\
\hline
\end{tabular}

a Разница в энергиях адсорбции на поверхности $\mathrm{Pt}(111)$ и кластере $\mathrm{Pt}_{79}{ }^{6}$ Центр адсорбции на поверхности платины (рис. 1).

$\mathrm{Pt}_{79}$ (центр top-corner) прочнее на 0,23 эВ по сравнению с адсорбцией на top-центре идеальной поверхности $\mathrm{Pt}(111)$. Мы полагаем, что более сильное взаимодействие с нанокластером обусловлено наличием НК атомов (с КЧ = 6) с большей подвижностью. Более выгодная адсорбция метанола (на 0,07 эВ) на top-центрах (КЧ = 7) ребра $\mathrm{Pt}(211)$ по сравнению с top-центрами идеальной поверхности $\operatorname{Pt}(111)$ была также найдена в работе [20].

Монооксид углерода. Адсорбция $\mathrm{CO}$ на поверхности $\mathrm{Pt}(111)$ хорошо изучена с помощью как экспериментальных, так и теоретических методов. Дифракция медленных электронов (ДМЭ) и спектроскопия характеристических потерь энергии электронов [38, 39] показали, что при молекулярном покрытии $<1 / 2 \mathrm{MC}$ молекула $\mathrm{CO}$ адсорбируется преимущественно на top-центрах поверхности $\mathrm{Pt}(111)$. При более высоком покрытии заселяются как top, так и bridge-центры. Определенная методом адсорбционной микрокалориметрии теплота адсорбции СО на $\mathrm{Pt}(111)$ уменьшалась от $-1,90 \pm 0,08$ эВ до -1,22 $\pm 0,20$ эВ при увеличении молекулярного покрытия СО [41].

Рассчитанные нами энергии адсорбции СО на центрах $f c c, h c p$, bridge и top поверхности $\operatorname{Pt}(111)$ составляют $-1,83,-1,81,-1,81$ и -1,73 эВ соответственно. Предсказанное наиболее стабильное адсорбционное положение $f c c$ не согласуется с экспериментально найденными положениями top или bridge, но согласуется с большинством расчетов, проведенных методом функционала плотности [41].

Найдено, что наиболее предпочтительные центры адсорбции СО на нанокластере $\mathrm{Pt}_{79}$ - вершина (top-corner) и мостиковое положение на ребре (bridge-edge) с энергиями адсорбции 2,19 и 2,28 эВ соответственно. Исследования методами просвечивающей электронной микроскопии и рентгеновской фотоэлектронной спектроскопии показали, что как bridge, так и top-конфигурации стабильны на нанокластерах [25]. Стабилизация энергии по сравнению с top- и bridge-центрами регулярной поверхности $\mathrm{Pt}(111)$ составляет до 
$33 \%(0,56$ эВ). При удалении от ребер и вершин к центру нанограней (111) стабилизация уменьшается.

Метоксигруппа. При разрыве связи О-Н в молекуле метанола образуется метоксигруппа $\mathrm{CH}_{3} \mathrm{O}$. Как и в случае метанола, взаимодействие с платиной происходит через атом кислорода. На поверхности платины $\mathrm{Pt}(111)$ существует три центра адсорбции для метоксигруппы: монокоординированный (top), двухкоординированный (bridge) и трехкоординированный $(f c c)$. Полученные энергии адсорбции составляют -1,80, -1,75 и -1,17 эВ соответственно. Таким образом, минимальная координация адсорбционного центра максимально выгодна энергетически. Поскольку в газовой фазе метоксигруппа имеет открытую электронную оболочку и представляет собой радикальную частицу, энергия её адсорбции оказывается достаточно высокой благодаря взаимодействию с электронами металла. Тем не менее в литературе отсутствуют данные о прямых спектроскопических наблюдениях метоксичастиц на поверхности платины, что, возможно, обусловлено довольно низким барьером разложения 0,25 эВ, рассчитанным в работе [17]. Рассчитанная нами энергия адсорбции, $-1,80$ эВ (top-центр), хорошо согласуется с рассчитанным значением -1,54 эВ для top-центра на гладкой поверхности $\operatorname{Pt}(111)$ [17]. Расчеты адсорбции $\mathrm{CH}_{3} \mathrm{O}$ при более плотном покрытии $(0,25 \mathrm{MC})$ показали, что наиболее предпочтительным является мостиковое положение с энергией -1,67 эВ [16]. Методом микрокалориметрии было определено более высокое значение энергии адсорбции на кислородсодержащей поверхности монокристалла $\mathrm{Pt}(111),-1,94 \pm 0,11$ эВ [42], обусловленое стабилизицией $\mathrm{CH}_{3} \mathrm{O}$ поверхностными ОН-группами. Полученная в работе [42] энергия адсорбции метанола, -0,61 эВ, также выше почти всех известных рассчитанных значений.

На нанокластере $\mathrm{Pt}_{79}$ метоксигруппа сохраняет связывание через атом кислорода. Найдено, что наиболее предпочтительными центрами адсорбции метоксигруппы являются bridgeedge - центры на стыке граней 111/100 (-2,37 эВ) и 111/111 (2,30 эВ). Энергия адсорбции в topcorner-центре составляет $-2,34$ эВ. Стабилизация $\mathrm{CH}_{3} \mathrm{O}$-частиц по сравнению с поверхностью $\operatorname{Pt}(111)$ достигает 35 \% (на 0,62 эВ), причем сильнее стабилизируются мостиковые комплексы. По-видимому, здесь большую роль играет расстояние между НК-атомами платины на ребрах частицы с координационным числами 6 и 6 (на стыке граней 111/100), 6 и 7 (на стыке граней 111/111), обладающими большей степенью свободы, чем атомы идеальной поверхности $\mathrm{Pt}(111)$ с КЧ, равным 9.

Анализ длин связей показал, что адсорбция $\mathrm{CH}_{3} \mathrm{O}$ приводит к значительному удлинению локальных связей Pt-Pt как на идеальной поверхности (до 0,13 $\AA$ ), так и на наночастице (до $0,28 \AA$ для самого стабильного комплекса bridge-edge 111/100 и до 0,35 $\AA$ при адсорбции на нанограни (111)). Вместе с тем взаимодействие $\mathrm{CH}_{3} \mathrm{O}$ с вершиной и ребром нанокластера 111/111 вызывает незначительное увеличение длин связей Pt-Pt только на $0,06 \AA$.

Формальдегид. Адсорбцию формальдегида трудно охарактеризовать экспериментально, поскольку он быстро разлагается на $\mathrm{CO}$ и $\mathrm{H}_{2}$. Кроме того, молекула $\mathrm{CH}_{2} \mathrm{O}$ имеет закрытые электронные оболочки, поэтому легко десорбируется с поверхности. Косвенно о присутствии адсорбированной формы формальдегида можно заключить на основании данных методов ДМЭ и атомной эмиссионной спектроскопии, с помощью которых был зафиксирован интермедиат, лежащий параллельно поверхности [43]. Рассчитанная нами энергия адсорбции $\mathrm{CH}_{2} \mathrm{O}$ на идеальной поверхности $\operatorname{Pt}(111)$ невысока, $-0,58$ эВ, даже в наиболее выгодном положении 
top-top, когда формальдегид ориентирован почти параллельно поверхности и бидентатно связан с нею через атомы О и С. Монодентатная адсорбция формальдегида через атом кислорода [44] в top-положении нестабильна и превращается в бидентатную форму top-top. Рассчитанная энергия адсорбции на поверхности $\mathrm{Pt}(111)$ хорошо согласуется с определенными методом ТПД экспериментальными значениями $-0,57$ и -0,72 эВ [45], -0,54 эВ [43], а также рассчитанными величинами $-0,50$ [16] и $-0,51$ эВ [17].

Мы рассмотрели различные бидентатные способы координации формальдегида на наночастице $\mathrm{Pt}_{79}$ и обнаружили, что наиболее прочно он адсорбируется на ребре наночастицы (с энергией $-1,28$ эВ), а наименее прочно - на грани нанокластера (с энергией -0,79 эВ). Положение, когда атом О связан с top-центром вершины, а атом $\mathrm{C}$ - с top-центром ребра (центр top-corner $(O)$-top-edge(C)), на 0,08 эВ более выгодно, чем инверсное (центр top-corner(C)-topedge(O)), когда атом С находится в вершине. Такая повышенная стабилизация (более чем в два раза) на ребрах наночастицы, как и в случае метоксигруппы, по-видимому, обусловлена большей способностью двух НК-атомов платины подстраиваться к длине связи С-О. Однако ориентация формальдегида на двух атомах платины с минимальным координационным числом 6 (top-corner $(O)$-top-corner $(C))$ на границе 111 и 100 плоскостей менее выгодна (1,09 эВ).

Формил. Несмотря на довольно прочную адсорбцию СНО на поверхности платины, его проблематично обнаружить экспериментально. Так, частоты колебания $\mathrm{C}=\mathrm{O}$, характерные для адсорбированного интермедиата НСО, наблюдали с помощью методов усиленного поверхностью рамановского рассеяния [46] и инфракрасной спектроскопии [47] при изучении разложения метанола и муравьиной кислоты на Аu- и Рt-электродах.

На поверхности $\mathrm{Pt}(111)$ нами найдены две возможные координации адсорбции СНО, очень близкие по энергии: одна, связанная с $t o p$-центром через атом $\mathrm{C}\left(\mathrm{E}_{\mathrm{ads}}=-2,52\right.$ эВ), и вторая, тридентатная bridge $(C)-t o p(O)\left(\mathrm{E}_{\mathrm{ads}}=-2,46\right.$ эВ), в которой атом водорода максимально отвернут от поверхности. Неудивительно, что в разных работах указывают на различные конфигурации адсорбции СНО как предпочтительные: тридентатную на центре $\operatorname{bridge}(C)-\operatorname{top}(O)$ с энергией $-2,46$ эВ [16] или монодентатную на top-центре с энергией $-2,36$ эВ [17]. В последнем случае разница энергий между двумя наиболее стабильными состояниями составила 0,12 эВ. Согласно расчетам на кластерной $\mathrm{Pt}_{10}$ модели поверхности $\mathrm{Pt}(111)$ монодентатная адсорбция $\mathrm{CHO}$ также является более стабильной с энергией $-2,60$ эВ [48].

Несмотря на близость значений рассчитанных энергий адсорбции комплексов СНО, длины связей С-О отличаются значительно. Рассчитанное для монодентатного комплекса расстояние С-О соответствует двойной связи (1,20 $)$, а для тридентатного - одинарной $(1,29 \AA)$. В некоторых экспериментальных исследованиях помимо характеристической частоты колебания связи С-О 1600-1700 см-1 указывается также более низкая частота: $\sim 1100-1200 \mathrm{~cm}^{-1}[8,46]$. Повидимому, более низкая частота колебания соответствует тридентатному комплексу.

На нанокластере $\mathrm{Pt}_{79}$ наиболее предпочтительным является бидентатное положение формильной группы на ребре top-corner(C)-top-edge(O) (с энергией -3,00 эВ). Инверсная конфигурация top-corner $(O)$-top-edge $(C)$ с атомом углерода в вершине менее стабильна на 0,04 эВ. Среди top-положений самое выгодное не на вершине (-2,74 эВ), а на грани кластера (2,81 эВ). В целом стабилизация на нанокластере по сравнению поверхностью платины достигает 0,48 эВ, что меньше, чем для метоксигруппы (0,62 эВ) и формальдегида $(0,70$ эВ).

$$
-437-
$$


Aтомарный водород. Полученные энергии адсорбции атомарного водорода на top-, bridge-, $f c c$ - и $h c p$-центрах поверхности $\mathrm{Pt}(111)$ составляют $-2,77,-2,73,-2,77$ и $-2,72$ эВ соответственно. Наиболее энергетически выгодна адсорбция на top- и $f c c$-центрах. Разница в энергиях адсорбции в пределах 0,05 эВ указывает на то, что при разрыве О-Н-связи атом водорода легко диффундирует по поверхности и, следовательно, может занять любой центр. Спектроскопия характеристических потерь энергии электронов, дифракция атомов Не, рассеяние низкоэнергетической отдачи, эксперименты по рассеянию нейтронов показывают предпочтительное $f c c$ заселение [49-51]. Рассчитанные нами энергии диссоциации молекулы $\mathrm{H}_{2}\left(H_{2}+2 * S \rightarrow 2 * H / S\right)$ для идеальной поверхности $\mathrm{Pt}(111),-0,90-0,99$ эВ, хорошо согласуются с экспериментальными данными [49-51], -0,70-0,83 эВ, полученными с помощью термодесорбционной микроскопии, и теоретическими данными, 0,88 эВ [52].

На нанокластере $\mathrm{Pt}_{79}$ атом водорода не оказывает особой предпочтительности НК-центрам. Ловушкой для водорода может служить top-положение на нанограни (111) и мостиковое bridgeedge на ребре с энергией адсорбции $-2,95$ эВ. Все остальные положения связывают водород с энергиями $-2,76-2,85$ эВ: сильнее на мостиковых центрах $(-2,85$ эВ) и слабее в top и $f c c$ $(-2,76$ эВ).

\section{Энергетический профиль реакиии}

На основании результатов расчетов наиболее энергетически выгодных адсорбционных комплексов анализируемых частиц был построен энергетический профиль реакции разложения метанола на регулярной поверхности $\mathrm{Pt}(111)$ и на кластере $\mathrm{Pt}_{79}$ (рис. 2). Для построения профиля были использованы не самые стабильные конфигурации $\mathrm{CH}_{3} \mathrm{O}$ (bridge-edge 111/100 c $\mathrm{E}_{\mathrm{ads}}=-2,37$ эВ) и $\mathrm{CHO}$ на $\mathrm{Pt}_{79}$ (top-corner(C)-top-edge $(O)$ с $\mathrm{E}_{\mathrm{ads}}=-3,00$ эВ), а следующие по стабильности изомеры top-corner $\left(\mathrm{E}_{\mathrm{ads}}=-2,34\right.$ эВ) и top-corner $(O)$-top-edge $(C)\left(\mathrm{E}_{\mathrm{ads}}=-2,95\right.$ эВ). $\mathrm{B}$ выбранных для построения энергетического профиля комплексах атом кислорода частицы $\mathrm{COH}_{x}$ связан с атомом Pt на вершине нанокластера. Было использовано допущение, что атом $\mathrm{H}$ не взаимодействует с другими участниками реакции. Дегидрирование метанола до $\mathrm{CH}_{3} \mathrm{O}$ на поверхности $\operatorname{Pt}(111)$ протекает с затратой энергии $\mathrm{E}_{\mathrm{R}}=0,50$ эВ, что является одной из причин нестабильности метоксигрупп. Рассчитанная энергия реакции дегидрирования $\mathrm{CH}_{3} \mathrm{OH}^{*}$ $\rightarrow \mathrm{CH}_{3} \mathrm{O}^{*}+\mathrm{H}^{*}$ на идеальной поверхности $\mathrm{Pt}(111)$ сравнима с теплотой реакции $0,59 \pm 0,1$ эВ, экспериментально определенной с помощью метода адсорбционной микрокалориметрии на монокристалле Pt(111) [42], а также с рассчитанным значением 0,61 эВ [17]. В то же время на кластере $\mathrm{Pt}_{79}$ близость к нулю энергии этой реакции повышает вероятность возникновения метоксигрупп и в целом протекания реакции по данному маршруту.

Для всех остальных стадий дегидрирования метанола на кластерах, за исключением десорбции СО, увеличивается экзотермический эффект на $\sim 0,2-0,5$ эВ.

С нашей точки зрения, реакция дегидрирования на нанокластере происходит следующим образом. Метанол адсорбируется в вершине нанокластера (top-corner-центр) кислородным концом. После разрыва О-Н-связи $\mathrm{CH}_{3} \mathrm{O}$-группа все еще остается в том же положении под углом к поверхности, ориентированная вдоль ребра (дефектного центра). Затем происходит образование второй связи с поверхностью через атом углерода (top-corner $(O)$-top-edge $(C)$ положение) и отрыв второго атома $\mathrm{H}$ с образованием формальдегида $\mathrm{CH}_{2} \mathrm{O}($ top-corner $(\mathrm{O})$ - 
top-edge $(C))$. Затем бидентатно связанная с поверхностью молекула $\mathrm{CH}_{2} \mathrm{O}$ может либо десорбироваться, либо потерять еще один атом водорода с образованием формильной группы $\mathrm{CHO}$, также бидентатно связанной с поверхностью (top-corner $(O)$-top-edge $(C))$. При отрыве последнего атома водорода остается СО либо в более стабильном мостиковом положении (-2,28 эВ), либо в угловом положении top-corner (-2,19 эВ). В последнем случае предполагается смещение $\mathrm{CO}$ с top-edge- на top-corner-центр. Миграция СО в top-edge-положение на ребре менее выгодна (-2,07 эВ).

На гладкой поверхности наблюдается более простая картина. Молекула $\mathrm{CH}_{3} \mathrm{OH}$, адсорбируясь на поверхности в tор-центре кислородным концом, наклоняется к поверхности. Образовавшаяся в результате потери атома водорода $\mathrm{CH}_{3} \mathrm{O}$-группа наклоняется к поверхности еще больше, пока не образуется вторая связь Pt-C с соседним top-центром. Последующее дегидрирование приводит к образованию СНО-группы, связанной с top-центром через атом углерода. В это время атом кислорода отрывается от поверхности и молекула как бы переворачивается. На последней стадии дегидрирования остается молекула СО, связанная с tор-центром. Из-за известной “проблемы правильного описания положения адсорбированной молекулы СО на платине” [41] в качестве референсной мы взяли не самую стабильную $f c c$-координацию, а topкоординацию, найденную экспериментально как наиболее предпочтительную.

\section{Заключение}

Методом функционала плотности изучены структура и энергии поверхностных комплексов, образующихся в реакции дегидрирования метанола по механизму разрыва связи O-Н на платиновом катализаторе. Найдено, что наиболее устойчивые комплексы образуются при адсорбции С- и О-содержащих интермедиатов на низкокоординированных атомах наночастицы $\mathrm{Pt}_{79}$. В то же время атомы $\mathrm{H}$, по данным расчётов, не проявляют избирательности при связывании с атомами металлического субстрата с различными координационными числами. Абсолютная энергия адсорбции на вершинах и ребрах нанокластера $\mathrm{Pt}_{79}$ выше на 0,2-0,7 эВ, чем на высококоординированных центрах регулярной поверхности $\mathrm{Pt}(111)$. Стабильность адсорбционных комплексов на поверхности нанокластера уменьшается от вершин к ребрам и затем к центру граней (111) нанокластера. Более высокая стабилизация адсорбатов на кластере вызвана большей подвижностью низкокоординированных атомов (со смещением атомов Pt до 0,35 ̊ на нанокластере по сравнению с таковым до $0,13 \AA$ на поверхности) и электронными эффектами нанокластера. Анализ энергетического профиля реакции показывает, что реакция образования ключевого для определения механизма реакции интермедиата (метоксигруппы) на кластере $\mathrm{Pt}_{79}$ термонейтральна, тогда как на поверхности $\operatorname{Pt}(111)$ эндотермический эффект составляет 0,5 эВ. Все остальные стадии дегидрирования метанола экзотермичны, причём на нанокластерах экзотермический эффект увеличивается на $\sim 0,2-0,5$ эВ (за исключением десорбции СО) по сравнению с идеальной поверхностью $\mathrm{Pt}(111)$. Таким образом, наши результаты хорошо согласуются с экспериментальным наблюдением $[22,25,26]$ о предпочтительности протекания реакции дегидрирования метанола на дефектных центрах поверхности платины. Для всех остальных стадий дегидрирования метанола на нанокластере, за исключением десорбции СО, экзотермический эффект увеличивается на $\sim 0,2-0,5$ эВ. 
Данная работа выполнена при поддержске проекта РФФИ, грант 16-33-00578. Авторы благодарят Сибирский суперкомпьютерный центр (2. Новосибирск) и Суперкомпьютерный комплекс МГУ им. М.В. Ломоносова [53] за предоставленные вычислительные ресурсы.

\section{Список литературы}

1. Song C. Fuel processing for low-temperature and high-temperature fuel cells. Challenges and opportunities for sustainable development in the 21st century. Catal. Today 2002. Vol. 77, P. 17-49.

2. Miller A. V., Kaichev V. V., Prosvirin I. P., Bukhtiyarov V. I. Mechanistic study of methanol decomposition and oxidation on Pt(111). J. Phys. Chem. C 2013. Vol. 117(16), P. 8189-8197.

3. Sexton B. A. Methanol decomposition on platinum (111). Surf. Sci. 1981. Vol. 102, P. 271281.

4. Liu Z., Sawada T., Takagi N., Watanabe K., Matsumoto Y. Reaction intermediates in the oxidation of methanol on a Pt(111)-(2x2)O surface. J. Chem. Phys. 2003. Vol. 119. P. 4879-4886.

5. Akhter S., White J. M. A static SIMS/TPD study of the kinetics of methoxy formation and decomposition on O/Pt(111). Surf. Sci. 1986. Vol. 167, P. 101-126.

6. Attard G. A., Chibane K., Ebert H. D., Parsons R. The adsorption and decomposition of methanol on Pt(110). Surf. Sci. 1989. Vol. 224, P. 311-326.

7. Gibson K. D., Dubois L. H. Step effects in the thermal decomposition of methanol on Pt(111). Surf. Sci. 1990. Vol. 233, P. 59-64.

8. Wang J., Masel. R. I. Carbon-oxygen bond scission during methanol decomposition on $(1 \times 1)$ platinum(110). J. Am. Chem. Soc. 1991. Vol. 113, P. 5850-5856.

9. Franaszczuk K., Herrero E., Zelenay P., Wieckowski A. A comparison of electrochemical and gas-phase decomposition of methanol on platinum surfaces. J. Phys. Chem. 1992. Vol. 96(13), P. 7.

10. Diekhoner L., Butler D. A., Baurichter A., Luntz A. C. Parallel Pathways in Methanol Decomposition on Pt(111). Surf. Sci. 1998. Vol. 409, P. 384-391.

11. Skoplyak O., Menning C.A., Barteau M.A., Chen J.G. Experimental and theoretical study of reactivity trends for methanol on $\mathrm{Co} / \mathrm{Pt}(111)$ and $\mathrm{Ni} / \mathrm{Pt}(111)$ bimetallic surfaces. J. Chem. Phys. 2007. Vol. 127, P. 114707.

12. Levis R. J., Jiang Z. C., Winograd N., Akhter S., White K. M. Methyl formation from methanol decomposition on $\operatorname{Pd}\{111\}$ and $\operatorname{Pt}\{111\}$. Catal. Lett. 1988. Vol. 11, P. 385-389.

13. Kua J., Goddard W. Oxidation of methanol on 2nd and 3rd row group VIII transition metals (Pt, Ir, Os, Pd, Rh, and Ru): Application to direct methanol fuel cells. J. Am. Chem. Soc. 1999. Vol. 121(47), P. 10928-10941.

14. Ishikawa Y., Liao M., Cabrera C. Oxidation of methanol on platinum, ruthenium and mixed Pt-M metals (M = Ru, Sn): A theoretical study. Surf. Sci. 2000. Vol. 463(1), P. 66-80.

15. Watanabe T., Ehara M., Kuramoto K., Nakatsuji H. Possible reaction pathway in methanol dehydrogenation on Pt and Ag surfaces/clusters starting from $\mathrm{O}-\mathrm{H}$ scission: Dipped adcluster model study. Surf. Sci. 2009. Vol. 603(4), P. 641-646.

16. Desai S., Neurock M., Kourtakis K. A Periodic Density Functional Theory Study of the Dehydrogenation of Methanol over Pt(111). J. Phys. Chem. B 2002. Vol. 106(10), P. 2559-2568.

17. Greeley J., Mavrikakis M. A First-Principles Study of Methanol Decomposition on $\operatorname{Pt}(111)$. J. Am. Chem. Soc. 2002. Vol. 124(24), P. 7193-7201.

$$
-440-
$$


18. Greeley J., Mavrikakis M. Competitive Paths for Methanol Decomposition on Pt (111). J. Am. Chem. Soc. 2004. Vol. 126(12), P. 3910-3919.

19. Okamoto Y., Sugino O., Mochizuki Y., Ikeshoji T., Morikawa Y. Comparative study of dehydrogenation of methanol at $\mathrm{Pt}(111) /$ water and $\mathrm{Pt}(111) /$ vacuum interfaces. Chem. Phys. Lett. 2003. Vol. 377(1-2), P. 236-242.

20. Cao D., Lu G.-Q., Wieckowski A., Wasileski S. A., Neurock M. Mechanisms of methanol decomposition on platinum: A combined experimental and ab initio approach. J. Phys. Chem. B 2005. Vol. 109(23), P. 11622-11633.

21. Hartnig C., Spohr E. The role of water in the initial steps of methanol oxidation on $\operatorname{Pt}(111)$. Chem. Phys. 2005. Vol. 319, P. 185-191.

22. Каичев В. В., Просвирин И. П., Бухтияров В. И. Разложения и окисление метанола на платине: исследование методами РФЭС и масс-спектрометрии in situ. Кинетика и Катализ. 2014. T. 55, C. 535-546.

23. Niu C., Jiao J., Xing B., Wang G., Bu X. Reaction mechanism of methanol decomposition on Pt-based model catalysts: a theoretical study. J. Comput. Chem. 2010. Vol. 31(10), P. 2023-37.

24. Housmanns T. H. M., Koper M. T. M. Methanol Oxidation on Stepped Pt $[\mathrm{n}(111) \times(110)]$ Electrodes: A Chronoamperometric Study. J. Phys. Chem. B 2003. Vol. 107, P. 8557-8567.

25. Ubago-Pérez R., Carrasco-Marín F., Moreno-Castilla C. Carbon-supported Pt as catalysts for low-temperature methanol decomposition to carbon monoxide and hydrogen. Appl. Catal. A 2004. Vol. 275, P. 119-126.

26. Imamura S., Higashihara T., Saito Y., Aritani H., Kanai H., Matsumura Y., Tsuda N. Decomposition of methanol on Pt-loaded ceria. Catal. Today 1999. Vol. 50, P. 369-380.

27. Perdew J. P., Wang Y. Accurate and simple analytic representation of the electron-gas correlation energy. Phys. Rev. B. 1992. Vol. 45(23), P. 1324-13249.

28. Kresse G., Furthmüller J. Efficiency of ab-initio total energy calculations for metals and semiconductors using a plane-wave basis set. Comput. Mater. Sci. 1996. Vol. 6(1), P. 15-50.

29. Kresse G., Hafner J. Ab initio molecular-dynamics simulation of the liquid-metal-amorphoussemiconductor transition in germanium. Phys. Rev. B 1994. Vol. 49, P. 14251-14269.

30. Blöchl P. E. Projector augmented-wave method. Phys. Rev. B 1994. Vol. 50(24), P. 1795317979 .

31. Kresse G., Joubert D. From ultrasoft pseudopotentials to the projector augmented-wave method. Phys. Rev. B 1999. Vol. 59(3), P. 1758-1775.

32. Monkhorst H.J., Pack J.D. Special points for Brillouin-zone integrations. Phys. Rev. B 1976. Vol. 13(12), P. 5188-5192.

33. Yudanov I. V., Sahnoun R., Neyman K. M., Rösch N. Metal nanoparticles as models of single crystal surfaces and supported catalysts: Density functional study of size effects for $\mathrm{CO} / \mathrm{Pd}(111)$. J. Chem. Phys. 2002. Vol. 117, P. 9887-9896.

34. Yudanov I. V., Genest A., Rösch N. DFT Studies of Palladium Model Catalysts: Structure and Size Effects. J. Cluster Sci. 2011. Vol. 22, P. 433-448.

35. Yudanov I. V., Matveev A. V., Neyman K. M., Rösch N. How the C-O Bond Breaks during Methanol Decomposition on Nanocrystallites of Palladium Catalysts. J. Am. Chem. Soc. 2008. Vol. 130, P. 9342-9352. 
36. CRC Handbook of Chemistry and Physics, 76th ed. New York: CRC Press, 1996. 504 p.

37. Villegas I., Weaver M. J. Infrared spectroscopy of model electrochemical interfaces in ultrahigh vacuum: Surface-cation solvation in the $\mathrm{Pt}(111) / \mathrm{K}^{+}-$methanol system. J. Chem. Phys. 1995. Vol. 103, P. 2295-2307.

38. Hopster H., Ibach H. Adsorption of CO on Pt(111) and Pt 6(111) $\times(111)$ studied by high resolution electron energy loss spectroscopy and thermal desorption spectroscopy. Surf. Sci. 1978. Vol. 77, P. 109-117.

39. Steininger H., Lehwald S., Ibach H. On the adsorption of CO on Pt(111). Surf. Sci. 1982. Vol. 123, P. 264-282.

40. Yeo Y. Y., Vattuone L., King D. A. Calorimetric heats for $\mathrm{CO}$ and oxygen adsorption and for the catalytic CO oxidation reaction on Pt\{111\}. J. Chem. Phys. 1997. Vol. 106, P. 392-402.

41. Feibelman P. J., Hammer B., Nørskov J. K., Wagner F., Scheffler M., Stumpf R., Watwe R., Dumesic J. The CO/Pt(111) Puzzle. J. Phys. Chem. B 2001. Vol. 105, P. 4018-4025.

42. Karp E. M., Silbaugh T. L., Crowe M. C., Campbell C. T. Energetics of adsorbed methanol and methoxy on Pt(111) by microcalorimetry. J. Am. Chem. Soc. 2012. Vol. 134, P. 20388-20395.

43. Abbas N. M., Madix R. The effects of structured overlayers of sulfur on the kinetics and mechanism of simple reactions on Pt(111): I. Formaldehyde decomposition. J. Appl. Surf. Sci. 1981. Vol. 7, P. 241-275.

44. Delbecq F., Sautet P. Low-Temperature Adsorption of Formaldehyde on a Pt(111) Surface. A Theoretical Study. Langmuir 1993. Vol. 9, P. 197-207.

45. Henderson M. A., Mitchell G. E., White J. M. The decomposition of formaldehyde on Pt(111): A TPD and HREELS study. Surf. Sci. 1987. Vol. 188, P. 206-218.

46. Zhang Y., Weaver M. J. Application of surface-enhanced Raman spectroscopy to organic electrocatalytic systems: decomposition and electrooxidation of methanol and formic acid on gold and platinum-film electrodes. Langmuir 1993. Vol. 9, P. 1397-1403.

47. Beden B., Juanto S., Leger J.M., Lamy C. Infrared spectroscopic study of the methanol adsorbates at a platinum electrode: Part III. Structural effects and behaviour of a polycrystalline surface. J. Electroanal. Chem. 1987. Vol. 238, P. 323-331.

48. Gomes J. R. B., Gomes J. A. N. F. Adsorption of the formyl species on transition metal surfaces. J. Electroanal. Chem. 2000. Vol. 483, P. 180-187.

49. Nieuwenhuys B. E. Influence of the surface structure on the adsorption of hydrogen on platinum, as studied by field emission probe-hole microscopy. Surf. Sci. 1976. Vol. 59, P. 430-446.

50. Christmann K. Interaction of hydrogen with solid surfaces. Surf. Sci. Rep. 1988. Vol. 9, P. 1-163.

51. Godbey D. J., Somorjai G. A. The adsorption and desorption of hydrogen and carbon monoxide on bimetallic Re-Pt(111) surfaces. Surf. Sci. 1988. Vol. 204, P. 301-318.

52. Papoian G., Nørskov J. K., Hoffmann R. A Comparative Theoretical Study of the Hydrogen, Methyl, and Ethyl Chemisorption on the Pt(111) Surface. J. Am. Chem. Soc. 2000. Vol. 122, P. 4129-4144.

53. Sadovnichy V., Tikhonravov A., Voevodin V., Opanasenko V. "Lomonosov": Supercomputing at Moscow State University. In Contemporary High Performance Computing: From Petascale toward Exascale (Chapman \& Hall/CRC Computational Science). USA, Boca Raton: CRC Press, 2013. 283$307 \mathrm{p}$. 